\title{
Self-Similarity in Transverse Intensity Distributions in the Farfield Diffraction Pattern of Radial Walsh Filters
}

\author{
P. Mukherjee and L. N. Hazra \\ Department of Applied Optics and Photonics, University of Calcutta, Acharya Prafulla Chandra Shiksha Prangan, JD-2, \\ Sector-III, Salt Lake, Kolkata 700098, India
}

Correspondence should be addressed to L. N. Hazra; lnhaphy@caluniv.ac.in

Received 30 April 2014; Revised 22 August 2014; Accepted 26 August 2014; Published 17 September 2014

Academic Editor: Xiushan Zhu

Copyright (C) 2014 P. Mukherjee and L. N. Hazra. This is an open access article distributed under the Creative Commons Attribution License, which permits unrestricted use, distribution, and reproduction in any medium, provided the original work is properly cited.

In a recent communication we reported the self-similarity in radial Walsh filters. The set of radial Walsh filters have been classified into distinct self-similar groups, where members of each group possess self-similar structures or phase sequences. It has been observed that, the axial intensity distributions in the farfield diffraction pattern of these self-similar radial Walsh filters are also self-similar. In this paper we report the self-similarity in the intensity distributions on a transverse plane in the farfield diffraction patterns of the self-similar radial Walsh filters.

\section{Introduction}

A self-similar object is exactly or approximately similar to a part of itself; that is, the whole has the same shape or structure as one or more of the parts. Self-similarity is a typical property of fractals [1]. Derived from radial Walsh functions [2-4], radial Walsh filters form a set of orthogonal phase filters that take on values either 0 or $\pi$ phase, corresponding to +1 or -1 value of the radial Walsh functions over the prespecified annular regions of the circular filter. They may be considered as binary zone plates $[5,6]$ and have wide range of applications [7-15]. The order of the Walsh filter is equal to the number of zeros crossing within the specified domain. Various orders of the Walsh filters having self-similar replicating phase sequences constitute a group. It has been shown that the axial intensity distributions in the farfield diffraction pattern of a pupil with these self-similar groups of filters are also self-similar [16].

Earlier studies on fractal zone plates and diffracting apertures [17-22] were mainly confined to the case of axial intensity distribution. As a prelude to the study of the three-dimensional light distribution in the farfield region, it is necessary to study the characteristics of the intensity distribution on the transverse plane in the presence of these filters.
In this paper, we report our investigations on the transverse intensity distribution on the farfield plane of a pupil with radial Walsh filters. It is noted that the transverse intensity distributions exhibited by the self-similar groups of radial Walsh filters are also self-similar.

The next section deals with the farfield diffraction pattern in the transverse plane. Section 3 deals with self-similarity in the transverse intensity distribution of the self-similar radial Walsh filters, followed by the concluding section.

\section{Farfield Diffraction Pattern in the Transverse Plane}

With reference to Figure 1, the complex amplitude distribution on a transverse plane located at $O^{\prime}$ in the farfield of an axially symmetric imaging system is given by $[23,24]$

$$
F(p)=\int_{0}^{1} f(r) J_{0}(p r) r d r
$$

where $f(r)$ is the circularly symmetric pupil function representing the complex amplitude distribution over the exit pupil at $E^{\prime} . r$ is the normalized radial distance of a point $A$ on the exit pupil from the optical axis. $r=A E^{\prime} / \rho_{\max }$, where $\rho_{\max }$ 


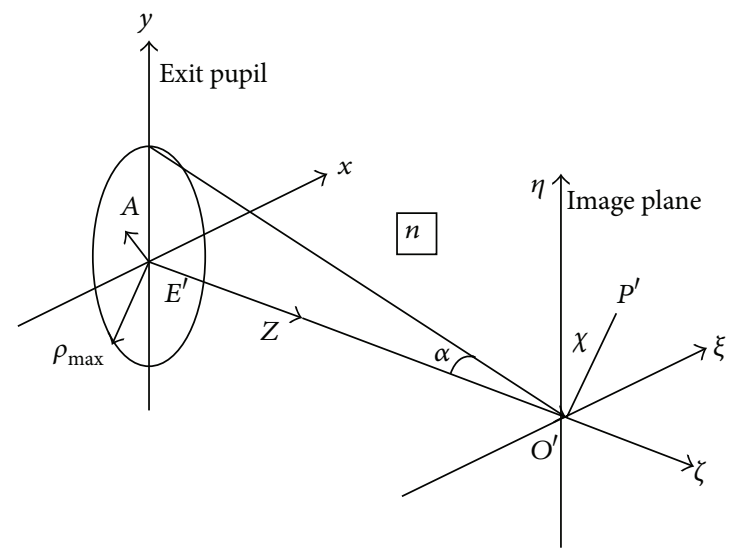

FIGURE 1: Exit pupil and image plane in the image space of an axially symmetric imaging system.

is the radius of the exit pupil. $p$ is the reduced diffraction variable defined as

$$
p=\frac{2 \pi}{\lambda}(n \sin \alpha) \chi
$$

where $\chi\left(=O^{\prime} P^{\prime}\right)$ is the geometrical distance of a point $P^{\prime}$ on the transverse farfield plane from the centre of the diffraction pattern. $(n \sin \alpha)$ is the image space numerical aperture. $k$, the propagation constant, is equal to $(2 \pi / \lambda)$, where $\lambda$ is the operating wavelength.

For an aberration-free pupil having uniform amplitude,

$$
f(r)= \begin{cases}1, & \text { over the aperture } \\ 0, & \text { elsewhere. }\end{cases}
$$

The normalized complex amplitude distribution on the transverse plane is

$$
F_{N}(p)=\frac{F(p)}{F(0)}=2 \int_{0}^{1} f(r) J_{0}(p r) r d r
$$

For a phase filter consisting of $M$ concentric equal area annular zones, place on the exit pupil of the imaging system, $f(r)$ may be expressed as

$$
f(r)=\sum_{m=1}^{M} f_{m} B_{m}(r)
$$

$B_{m}(r)$ are radial Walsh block functions defined as

$$
B_{m}(r)= \begin{cases}1, & r_{m-1} \leq r \leq r_{m} \\ 0, & \text { otherwise }\end{cases}
$$

where

$$
r_{m}=\left[\frac{m}{M}\right]^{1 / 2}, \quad r_{m-1}=\left[\frac{m-1}{M}\right]^{1 / 2} .
$$

The normalized complex amplitude distributions on the transverse plane is

$$
\begin{aligned}
F_{N}(p) & =2 \sum_{m=1}^{M} f_{m} \int_{0}^{1} B_{m}(r) J_{0}(p r) r d r \\
& =2 \sum_{m=1}^{M} f_{m} \int_{r_{m-1}}^{r_{m}} J_{0}(p r) r d r=2 \sum_{m=1}^{M} f_{m} \mathscr{J}_{m}(p),
\end{aligned}
$$

where

$$
\mathscr{J}_{m}(p)=\left[\frac{r_{m} J_{1}\left(p r_{m}\right)-r_{m-1} J_{1}\left(p r_{m-1}\right)}{p}\right]
$$

For a phase filter having the same phase $k \psi_{m}$ over the $m$ th zone

$$
f_{m}=\exp \left[i k \psi_{m}\right]
$$

From (8) and (10),

$$
F_{N}(p)=2 \sum_{m=1}^{M} \exp \left[i k \psi_{m}\right] \mathscr{J}_{m}(p) .
$$

The normalized intensity distribution on the transverse plane is

$$
I_{N}(p)=4 \sum_{m=1}^{M} \sum_{u=1}^{M}\left[\cos \left\{\left(k \psi_{m}\right)-\left(k \psi_{u}\right)\right\}\right] \mathscr{J}_{m}(p) \mathscr{J}_{u}(p)
$$

\section{Self-Similarity in Transverse Intensity Distributions of Self-Similar Radial Walsh Filters}

As discussed in detail in our previous communication [16], distinct groups of Walsh filters demonstrate self-similarity among their constituents. Groups were divided into subgroups and members of each subgroup were expressed by 

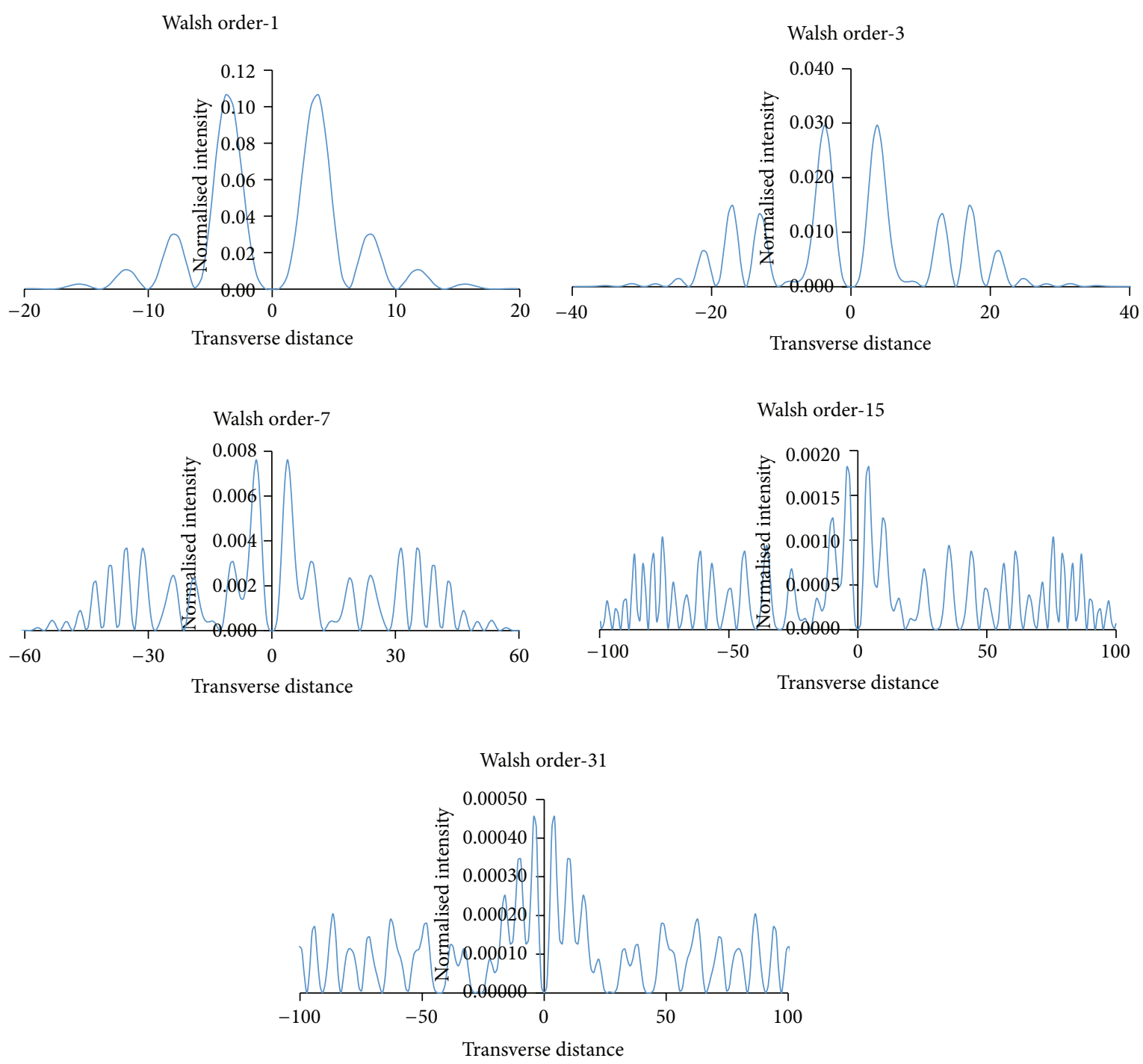

FiguRE 2: Transverse intensity distribution curves for radial Walsh filters of Group I, orders 1, 3, 7, 15, and 31.

an analytical expression $Z(\sigma)$. Details of the genesis of the subgroups were also discussed. On the basis of this classification the transverse intensity distribution curves for some of the groups of self-similar Walsh filters are put forward in this section. Our studies are mostly limited to Walsh orders $k$ within the range $(0,31)$. It has been observed that each group or more precisely each subgroup has unique self-similar transverse intensity patterns exhibited by its members.

Radial Walsh filters of Group I, that is, orders 1, 3, 7, 15, and 31, produce self-similar transverse intensity distribution. Orders 1 and 3 exhibit distinct dual maxima around the point $O^{\prime}$ along the transverse direction and fainter side lobes as illustrated in Figure 2. As we move from lower to higher orders, the two peaks on either side of the origin decrease in intensity and keep breaking into secondary and tertiary peaks. The number of fainter side lobes increases and their intensity decreases. However, the central peaks with their subpeaks maintain an intensity level much higher than the fainter side lobes. The horizontal change in scale in the transverse intensity distributions of the various members in Figure 2 was made to accommodate the two central lobes of highest intensity and few side lobes in the figure. Similar explanations hold for the horizontal scale change in the transverse intensity distributions for the various members of the other subgroups.

Subgroup IIA members are radial Walsh filters of orders 2 , 4,8 , and 16. Order 2 exhibits transverse intensity distribution with dual maxima and adjacent fainter side lobes as illustrated in Figure 3(a). Unlike Group I, as we move from lower to higher orders, the two central peaks on either side of the origin decrease in intensity and the side lobes shoot up in intensity to comparable scale as that of the central lobes. However, the number of side ripples keep increasing with increase in order.

As with subgroup IIA, the general trend observed for each subgroup is that, with increase in order of filters within a particular subgroup, as we move from lower to higher orders the side ripples increase in number and the intensity of the 
Walsh order-2

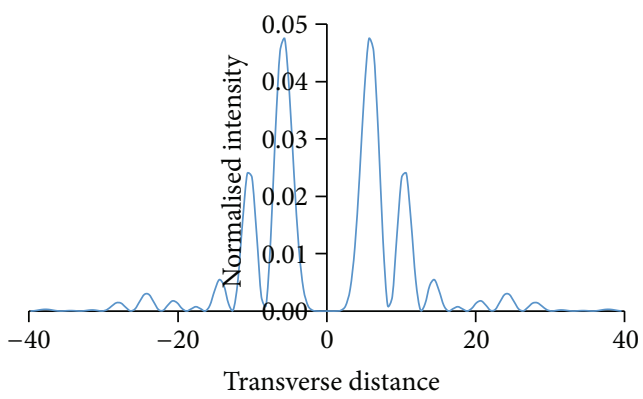

Walsh order-8

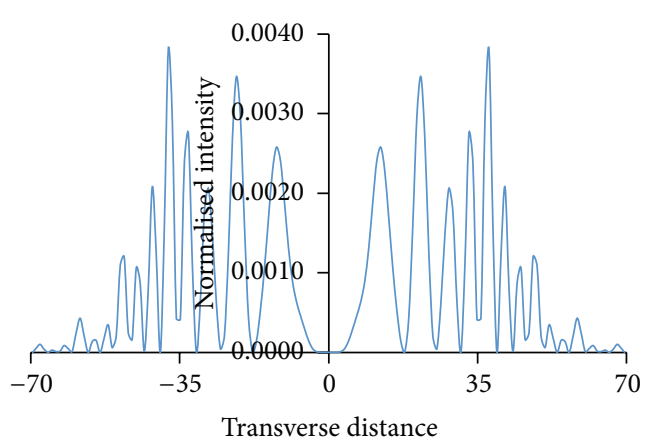

Walsh order-6

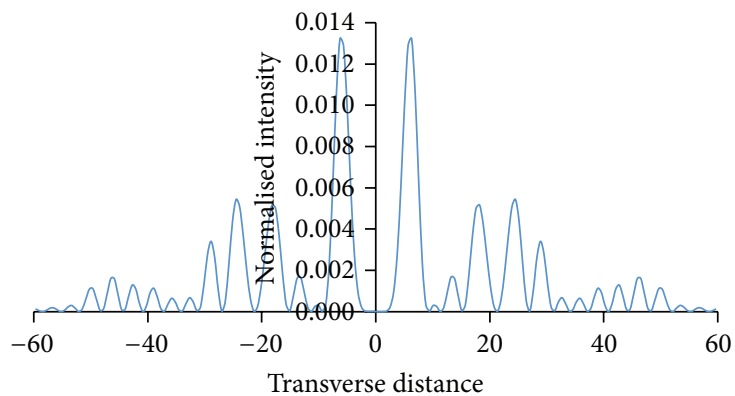

Transverse distance

(a)
Walsh order-4

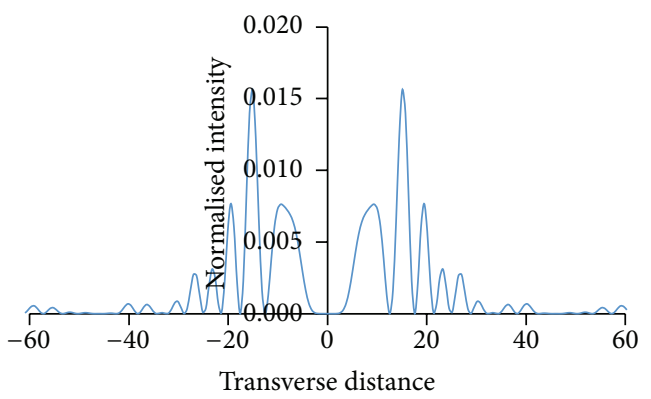

Walsh order-16

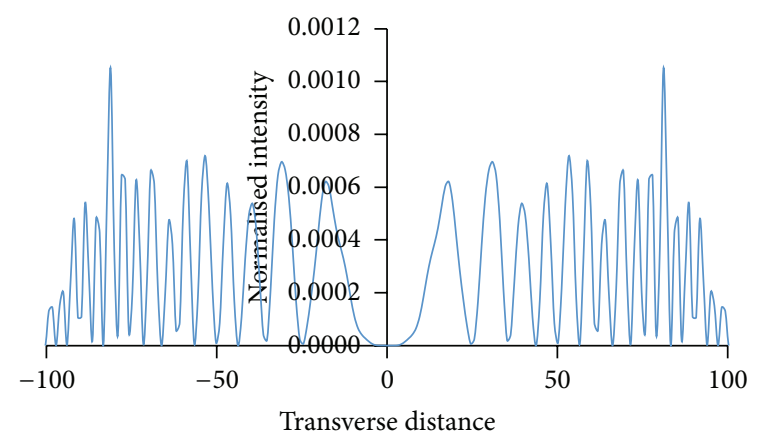

Walsh order-12

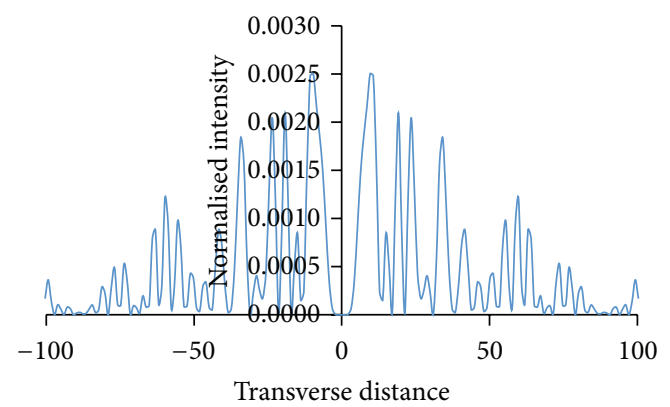

Walsh order-24

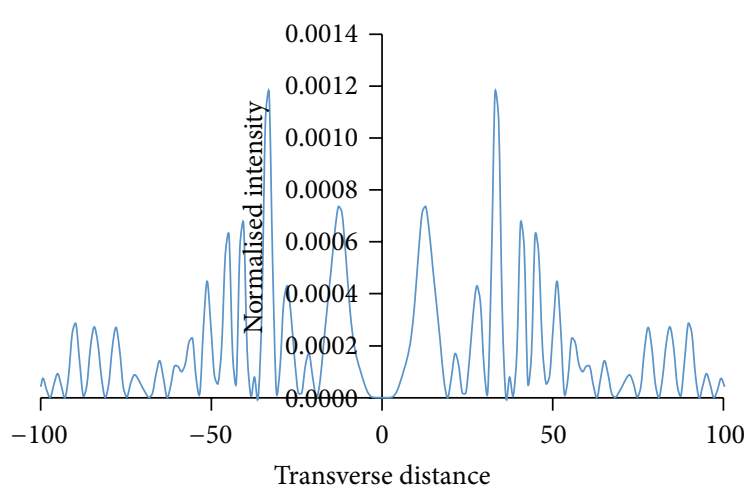

(b)

FIGURE 3: Continued. 

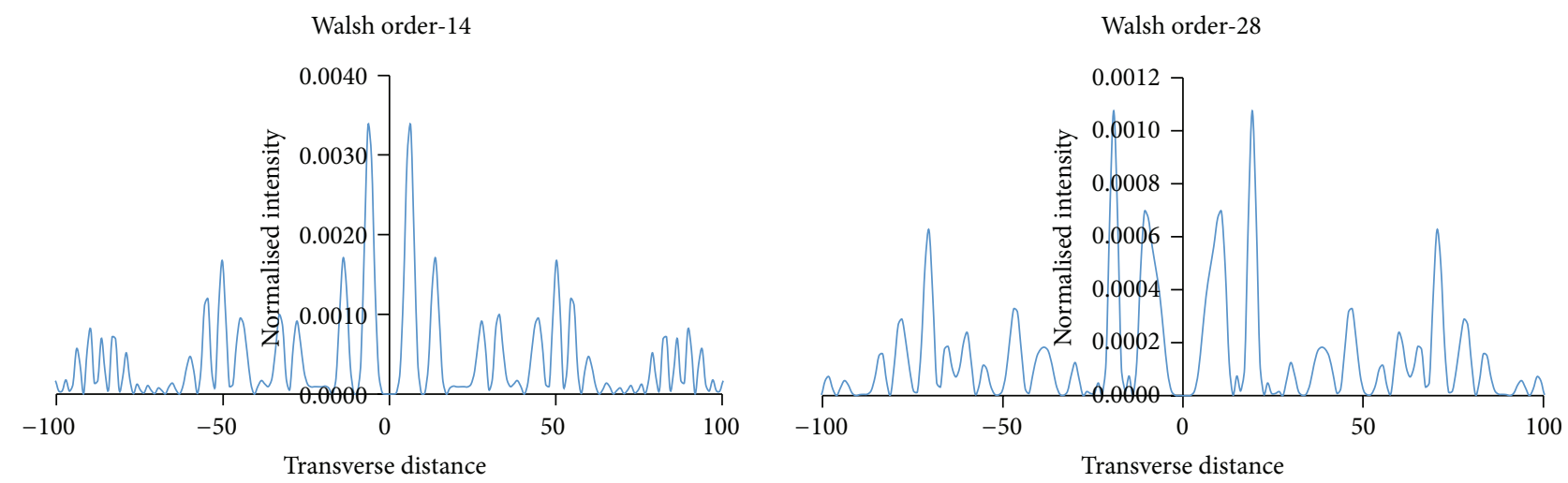

(c)

Figure 3: (a) Transverse intensity distribution curves for radial Walsh filters of Group IIA, orders 2, 4, 8, and 16. (b) Transverse intensity distribution curves for radial Walsh filters of Group IIB, orders 6, 12, and 24. (c) Transverse intensity distribution curves for radial Walsh filters of Group IIC, orders 14 and 28.

lobes near the origin and side ripples fall in magnitude. The decrease in intensity for the central lobes near the origin is more compared to the side lobes with the consequence that the side lobes shoot up in intensity to match the scale of the central lobe of that particular order. However, each subgroup has a unique self-similar pattern exhibited by its members.

Subgroup IIB contains Walsh orders 6, 12, and 24. These filters produce self-similar transverse intensity distributions with dual maxima pair placed on either side of the origin and side ripples in the form of triplet pairs placed symmetrically on either side of the dual maxima as illustrated in Figure 3(b). With increasing order of the filters, the transverse intensity patterns follow the general trend.

Subgroup IIC contains Walsh orders 14 and 28. These filters produce self-similar transverse intensity distributions as illustrated in Figure 3(c). The distribution consists of dual maxima and side lobe attached to the maxima just on either side of the origin. The ripples on either side of the maxima are comprised of faint dual and triplet intensity pairs, respectively, from the maxima, on either side of the origin. With increasing order the patterns follow the general trend and the side ripples increase in number to destroy the exact dual and triplet nature of the intensity distribution of the ripples.

Members of Group IIIAA, that is, radial Walsh filters of orders 5,11 , and 23 , produce transverse intensity distribution curves as illustrated in Figure 4(a). The pattern includes dual maxima on either side of the origin with fainter ripples of almost equal intensity.

Subgroup IIIAB contains Walsh orders 13 and 27. The self-similar transverse intensity distributions produced by these orders are depicted in Figure 4(b). The pattern has dual maxima placed on either side of the origin. The side ripples, respectively, from this maximum on either side of the origin consist of a triplet and a dual intensity distribution. Ripples are found between the dual and the triplet in the lowest order 13. With increasing orders the general trend is followed.
Subgroup IIIBA contains Walsh filter of orders 9 and 19. These orders produce self-similar transverse intensity distribution with higher side lobes as compared to the two central lobes on either side of the origin, as illustrated in Figure 4(c).

Walsh orders contained in subgroup IVAAA, that is, 10 and 20, produce self-similar transverse intensity distribution as illustrated in Figure 5. The patterns depict dual maxima on either side of the origin with side lobes. Triplets and doublets are seen just adjacent to the side lobe of the central lobe, followed by fainter side ripples. Unlike Subgroup IIIAB member 13, no ripples are found between the dual and the triplet in the lowest order 10.

It may be noted in Figures 2-5 that within each subgroup the intensity profiles are self-similar, which is a consequence of the inherent self-similarity in members of that subgroup. It may also be noted from these figures that, with increasing member order within a subgroup, the lobes become narrower with decreasing intensity and the distance between them increases. This is a general trend observed in the transverse intensity distributions of members of a subgroup.

Some studies were carried out on other subgroups whose first member falls within the range $(0,31)$ of $k$. It was observed that subgroups containing orders 17, 18, 21, 22, 25, 26 29 , and 30 are members of subgroups containing higher order Walsh filters. They produce transverse intensity distributions with slight variations from the patterns mentioned above and illustrated in Figures 2-5.

\section{Concluding Remarks}

The focusing properties of radial Walsh filters both in the axial and in the transverse directions portray self-similarity which can be correlated to the self-similar structures of the diffracting apertures, a characteristic exhibited by other fractal zone plates and diffracting apertures. This feature may be exploited in practice to produce complex $3 \mathrm{D}$ light distribution near the focus which finds application in $3 \mathrm{D}$ imaging, lithography, optical superresolution, optical micromanipulation, and optical tomography, to mention a few. 
Walsh order-5

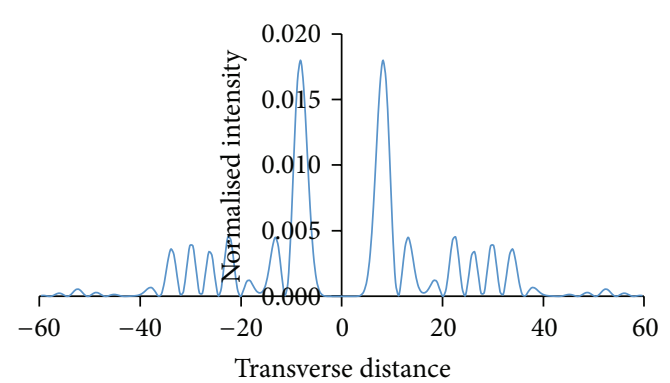

Walsh order-11

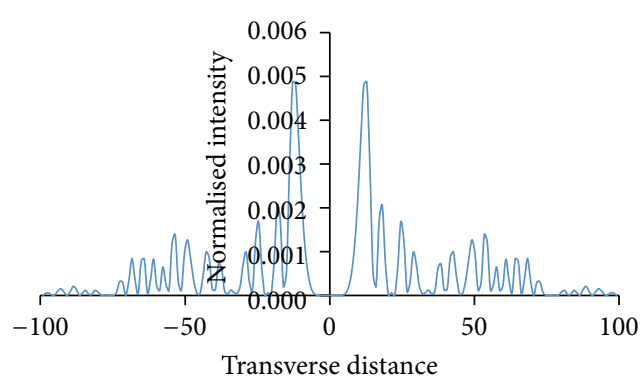

Walsh order-23

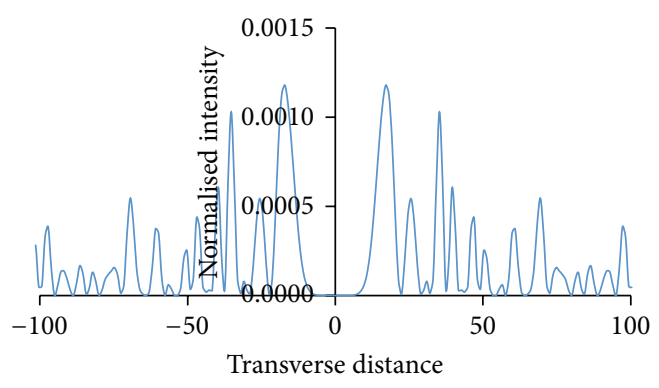

(a)

Walsh order-13

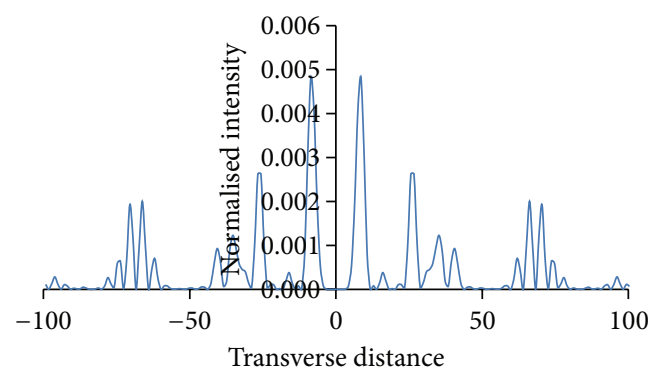

(b)
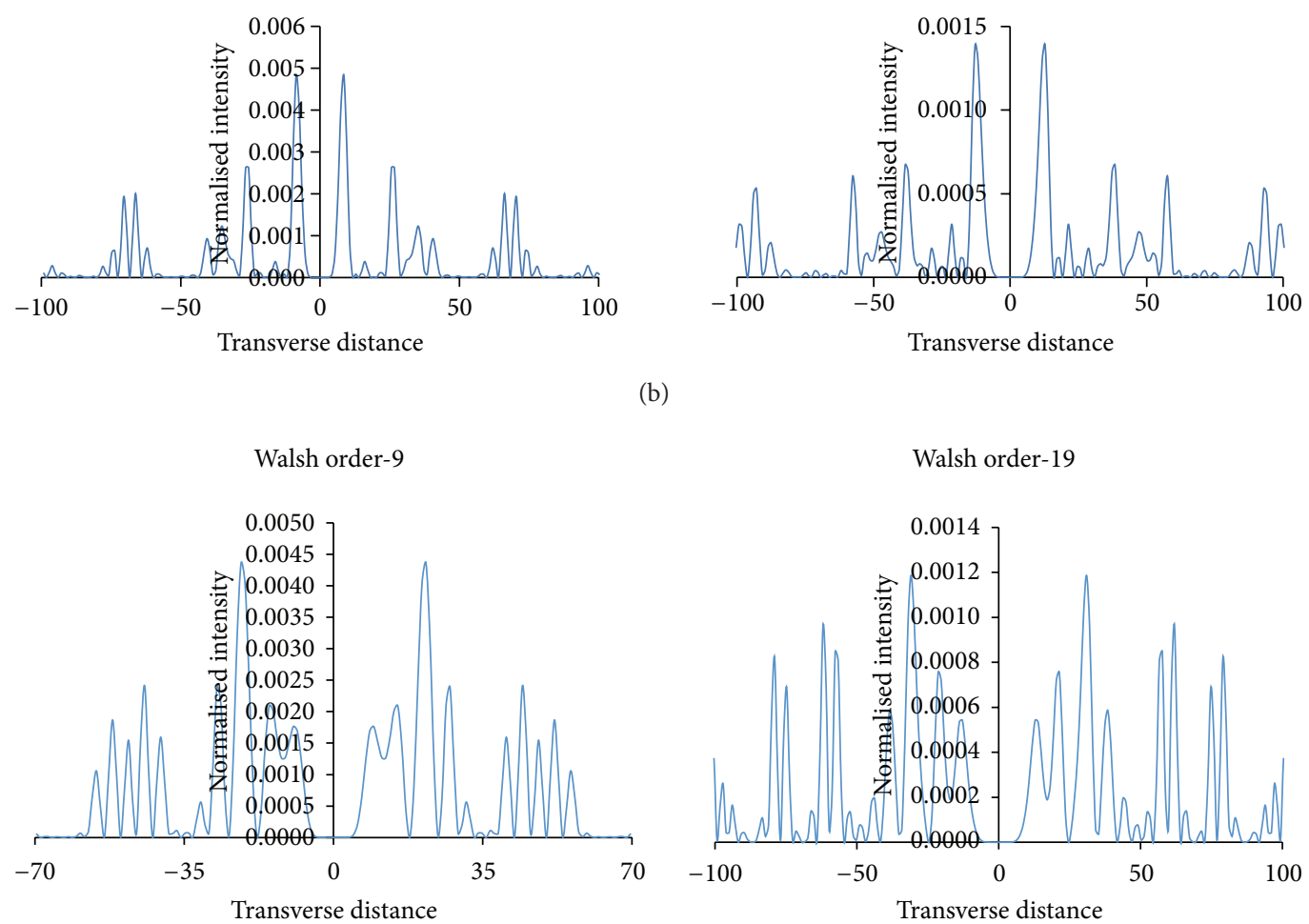

(c)

Figure 4: (a) Transverse intensity distribution curves for radial Walsh filters of Group IIIAA, orders 5, 11, and 23. (b) Transverse intensity distribution curves for radial Walsh filters of Group IIIAB, orders 13 and 27. (c) Transverse intensity distribution curves for radial Walsh filters of Group IIIBA, orders 9 and 19. 

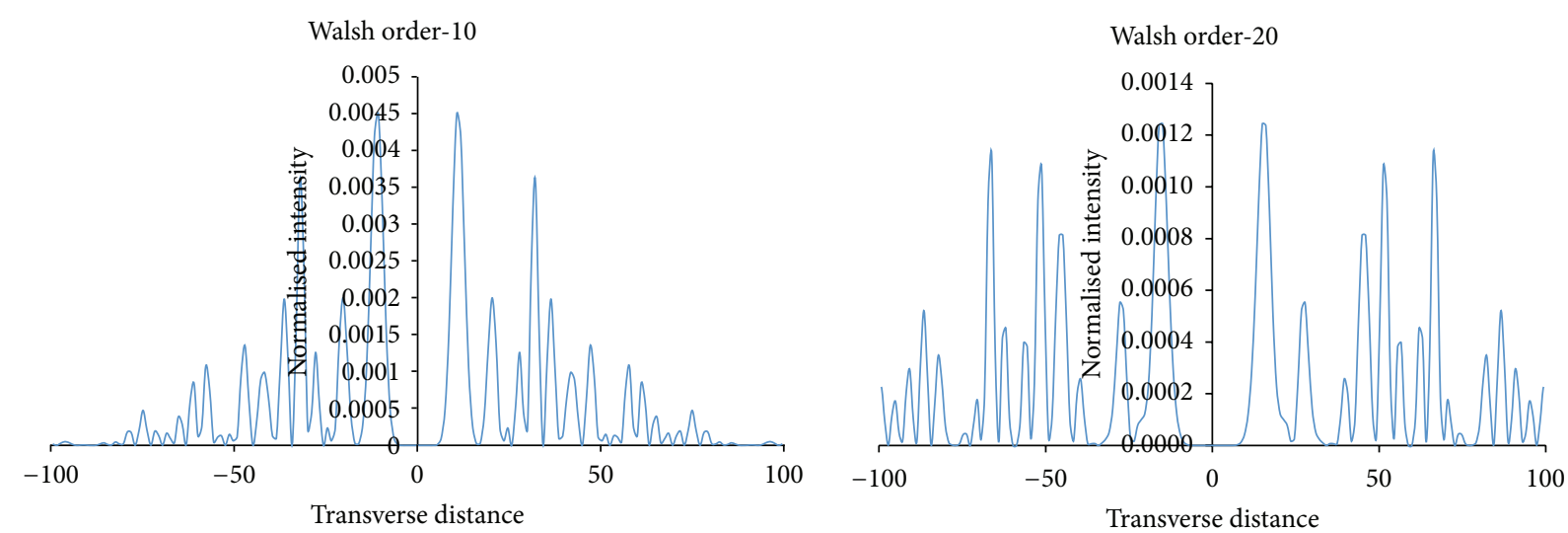

FIGURE 5: Transverse intensity distribution curves for radial Walsh filters of Group IVAAA, orders 10 and 20.

The inverse problem where a phase filter needs to be synthesized in accordance with a prespecified transverse intensity profile may be solved using the self-similar property of the energy efficient radial Walsh filters.

\section{Conflict of Interests}

The authors declare that there is no conflict of interests regarding the publication of this paper.

\section{References}

[1] B. B. Mandelbrot, The Fractal Geometry of Nature, W. H. Freeman, San Francisco, Calif, USA, 1982.

[2] J. L. Walsh, "A Closed Set of Normal Orthogonal Functions," A closed set of normal orthogonal functions, vol. 45, no. 1, pp. 5-24, 1923.

[3] H. F. Harmuth, Transmission of Information by Orthogonal Functions, Springer, Berlin, Germany, 1972.

[4] K. G. Beauchamp, Walsh Functions and Their Applications, Academic Press, New York, NY, USA, 1985.

[5] L. N. Hazra, Y. Han, and C. Delisle, "Stigmatic imaging by zone plates: a generalized treatment," Journal of the Optical Society of America A, vol. 10, no. 1, pp. 69-74, 1993.

[6] L. N. Hazra, Y. Han, and C. Delisle, "Imaging by zone plates: axial stigmatism at a particular order," Journal of the Optical Society of America A, vol. 11, no. 10, pp. 2750-2754, 1994.

[7] M. Fu, G. Wade, J. Ning, and R. Jakobs, "On walsh filtering method for decoding of CPM signals," IEEE Communications Letters, vol. 8, no. 6, pp. 345-347, 2004.

[8] H. C. Andrews, Computer Techniques in Image Processing, Academic, New York, NY, USA, 1970.

[9] L. N. Hazra and A. Banerjee, "Application of Walsh function in generation of optimum apodizers," Journal of Optics, vol. 5, pp. 19-26, 1976.

[10] M. De and L. N. Hazra, "Walsh functions in problems of optical imagery," Optica Acta, vol. 24, no. 3, pp. 221-234, 1977.

[11] M. De and L. N. Hazra, "Real-time image restoration through Walsh filtering," Optica Acta, vol. 24, no. 3, pp. 211-220, 1977.

[12] L. N. Hazra, "A new class of optimum amplitude filters," Optics Communications, vol. 21, no. 2, pp. 232-236, 1977.
[13] L. N. Hazra and A. Guha, "Farfield diffraction properties of radial Walsh filters," JOSA A, vol. 3, no. 6, pp. 843-846, 1986.

[14] L. N. Hazra, "Walsh filters for tailoring of resolution in microscopic imaging," Micron, vol. 38, no. 2, pp. 129-135, 2007.

[15] P. Mukherjee and L. Hazra, "Far-field diffraction properties of annular walsh filters," Advances in Optical Technologies, vol. 2013, Article ID 360450, 6 pages, 2013.

[16] P. Mukherjee and L. N. Hazra, "Self-similarity in radial Walsh filters and axial intensity distributions in the far-field diffraction pattern," Journal of the Optical Society of America A, vol. 31, no. 2, pp. 379-387, 2014.

[17] J. A Monsoriu, W. D. Furlan, and G. Saavedra, "Focussing light with fractal zone plates," in Recent Research Developments in Optics, vol. 5, 2005.

[18] G. Saavedra, W. D. Furlan, and J. A. Monsoriu, "Fractal zone plates," Optics Letters, vol. 28, no. 12, pp. 971-973, 2003.

[19] L. Zunino and M. Garavaglia, "Fraunhofer diffraction by Cantor fractals with variable lacunarity," Journal of Modern Optics, vol. 50, no. 5, pp. 717-727, 2003.

[20] J. Uozumi, Y. Sakurada, and T. Asakura, "Fraunhofer diffraction from apertures bounded by regular fractals," Journal of Modern Optics, vol. 42, no. 11, pp. 2309-2322, 1995.

[21] A. Calatayud, V. Ferrando, F. Giménez, W. D. Furlan, G. Saavedra, and J. A. Monsoriu, "Fractal square zone plates," Optics Communications, vol. 286, no. 1, pp. 42-45, 2013.

[22] V. Ferrando, A. Calatayud, F. Giménez, W. D. Furlan, and J. A. Monsoriu, "Cantor dust zone plates," Optics Express, vol. 21, no. 3, pp. 2701-2706, 2013.

[23] M. Born and E. Wolf, Principles of Optics, Pergamon Press, Oxford, UK, 1980

[24] H. H. Hopkins, "Canonical and real space coordinates used in the theory of image formation," in Applied Optics and Optical Engineering, R. R. Shannon and J. C. Wyant, Eds., vol. 9, pp. 307369, Academic, New York, NY, USA, 1983. 

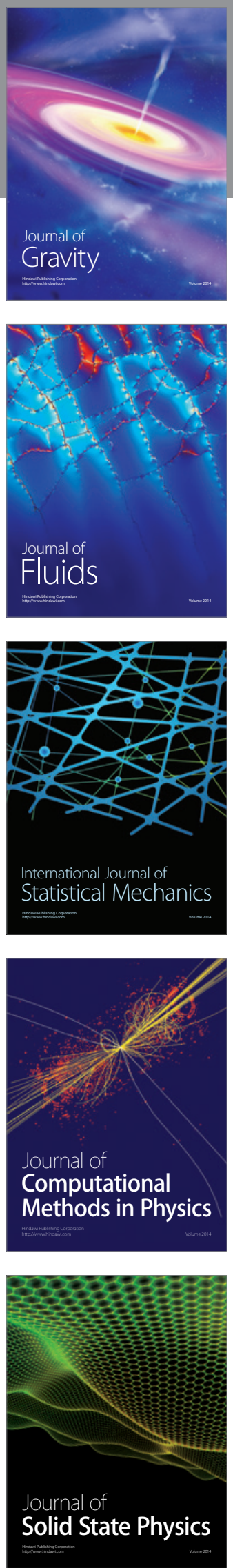

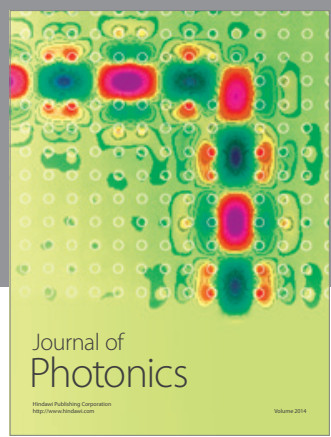

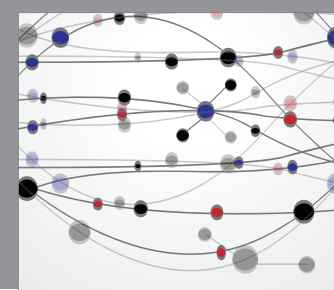

The Scientific World Journal

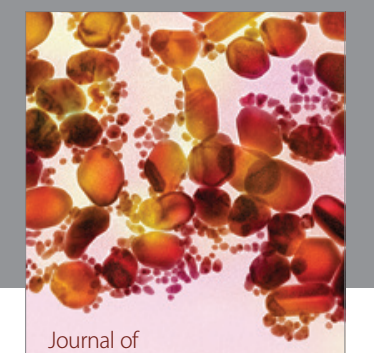

Soft Matter
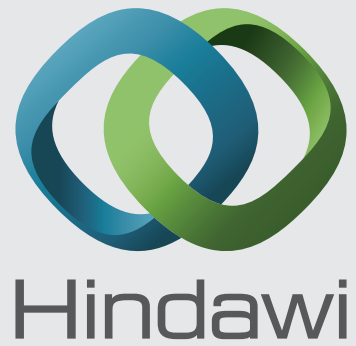

Submit your manuscripts at

http://www.hindawi.com
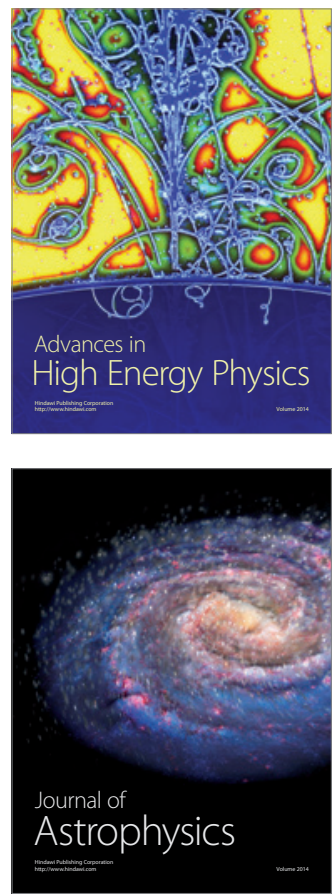
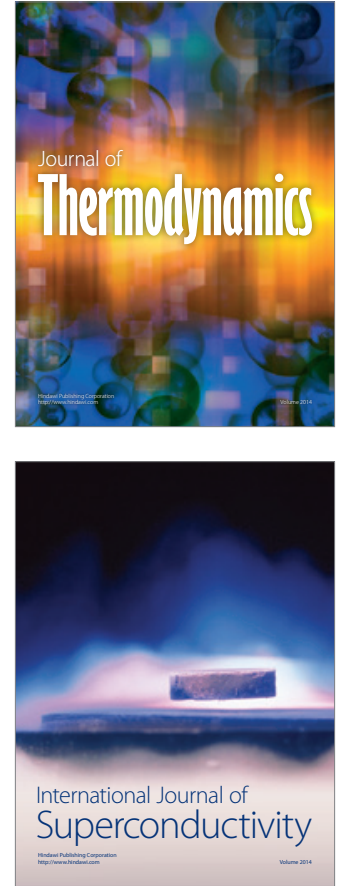
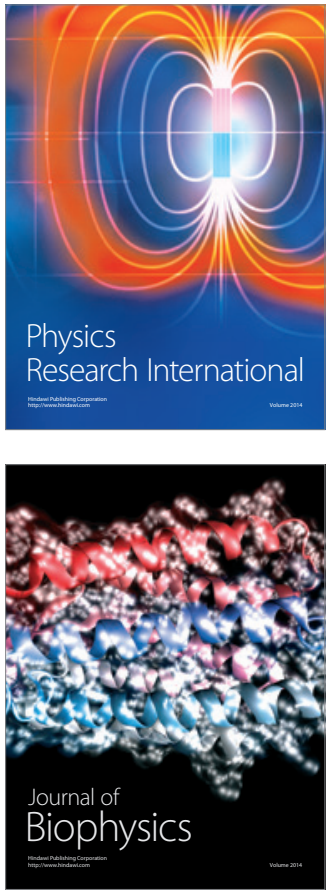
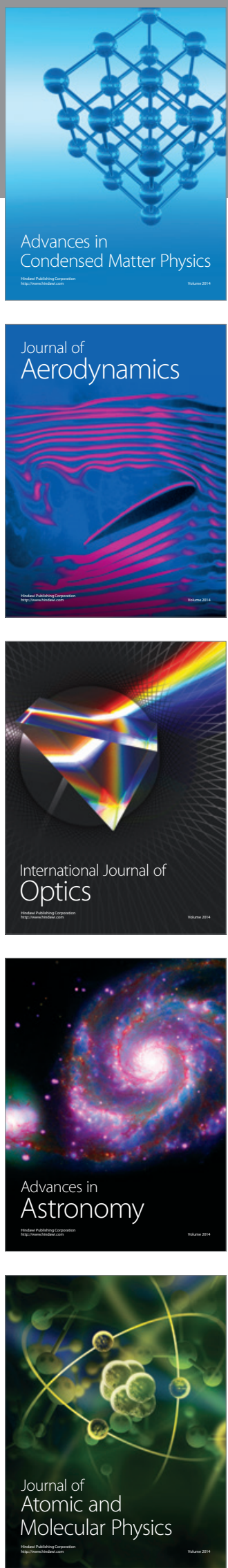